\title{
COLLECTIVE EFFECTS IN THE CLIC DAMPING RINGS
}

\author{
F. Zimmermann, M. Korostelev, D. Schulte, CERN, Geneva, Switzerland; \\ T. Agoh, K. Yokoya, KEK, Tsukuba, Japan
}

\section{Abstract}

The small emittance, short bunch length, and high current in the CLIC damping ring could give rise to collective effects which degrade the quality of the extracted beam. In this paper, we survey a number of possible instabilities and estimate their impact on the ring performance. The effects considered include fast beam-ion instability, coherent synchrotron radiation, Touschek scattering, intrabeam scattering, resistive-wall wake fields, and electron cloud.

\section{INTRODUCTION}

The design parameters of the CLIC damping ring [1] considered in this paper [2] are summarized in Table 1. The limitations encountered at storage rings with similar features are manifold, ranging from microwave instability (SLC DR), over ion effects (SLC DR, ATF, KEKB, PEP-II), electron cloud (KEKB, PEP-II, BEPC, CESR, DAFNE), intrabeam scattering (ATF), and transverse mode coupling (LEP), to longitudinal (ATF) and transverse coupled-bunch instabilities (KEKB, DAFNE).

Table 1: CLIC Damping-Ring Parameters

\begin{tabular}{|lcc|}
\hline variable & symbol & value \\
\hline bunch population & $N_{b}$ & $3 \times 10^{9}$ \\
bunch spacing & $L_{\text {sep }}$ & $20 \mathrm{~cm}$ \\
bunches per train & $n_{b}$ & 154 \\
no. of trains & $n_{\text {train }}$ & 9 \\
store time / train & $t_{\text {store }}$ & $90 \mathrm{~ms}$ \\
rms bunch length & $\sigma_{z}$ & $1.3 \mathrm{~mm}$ \\
rms momentum spread & $\sigma_{\delta}$ & $0.13 \%$ \\
final hor. emittance & $\gamma \epsilon_{x}$ & $578 \mathrm{~nm}$ \\
final vert. emittance & $\gamma \epsilon_{y}$ & $8.1 \mathrm{~nm}$ \\
circumference & $C$ & $357 \mathrm{~m}$ \\
beam pipe radius in arc & $b_{\text {arc }}$ & $2 \mathrm{~cm}$ \\
vertical half gap in wiggler & $b_{w}$ & $8 \mathrm{~mm}$ \\
no. of arc bends & $n_{\text {bend }}$ & 96 \\
arc-dipole field & $B_{\text {bend }}$ & $0.932 \mathrm{~T}$ \\
length of arc bend & $l_{\text {bend }}$ & $0.545 \mathrm{~m}$ \\
no. of wigglers & $n_{\text {wiggler }}$ & 76 \\
wiggler field & $B_{\text {wiggler }}$ & $1.764 \mathrm{~T}$ \\
length of wiggler & $l_{\text {wiggler }}$ & $2.1 \mathrm{~m}$ \\
momentum compaction & $\alpha_{c}$ & $0.731 \times 10^{-4}$ \\
synchrotron tune & $Q_{s}$ & 0.005 \\
integer betatron tunes & $Q_{x, y}$ & 72,34 \\
rf frequency & $f_{r f}$ & $1.5 \mathrm{GHz}$ \\
energy loss / turn & $U_{0}$ & $2.192 \mathrm{MeV}$ \\
rf voltage & $V_{r f}$ & $3 \mathrm{MV}$ \\
beam energy & $E_{b}$ & $2.424 \mathrm{GeV}$ \\
damping rime & $\tau_{s}$ & $1.32 \mathrm{~ms}$ \\
\hline & & \\
\hline
\end{tabular}

\section{LONGITUDINAL $\boldsymbol{\mu}$-WAVE INSTABILITY}

For $b>\sigma_{z}$, the Keil-Schnell-Boussard threshold is [3]

$$
\frac{Z}{n}=Z_{0} \sqrt{\frac{\pi}{2}} \frac{\gamma \alpha_{C} \sigma_{\delta}^{2} \sigma_{z}}{N_{b} r_{e}}\left(\frac{b}{\sigma_{z}}\right)^{2} \approx 3 \Omega,
$$

but it would be only $43 \mathrm{~m} \Omega$ without the suppression factor $\left(b / \sigma_{z}\right)^{2}$, where the beam-pipe radius $b \approx 11 \mathrm{~mm}$ represents a weighted average for arcs and wigglers. For comparison the KEKB LER ring has a design longitudinal impedance of $Z / n \approx 15 \mathrm{~m} \Omega$, while a much larger impedance of $Z / n \approx 72 \mathrm{~m} \Omega$ was measured [4]. Linear scaling would give $130 \mathrm{~m} \Omega$ or $625 \mathrm{~m} \Omega$, respectively, at the CLIC revolution frequency. This number is well below the above threshold estimate.

\section{TRANSVERSE MODE COUPLING}

The transverse TMC instability was the main intensity limitation in LEP. The instability threshold in a 2-particle model is reached for an impedance

$$
Z_{\perp, t h r} \approx \frac{8 \gamma Q_{\beta} Q_{s} b Z_{0}}{N r_{e} C} \approx 9 \mathrm{M} \Omega \mathrm{m}^{-1}
$$

corresponding to an equivalent $Z / n \approx\left(\pi b^{2} / C\right) Z_{\perp, t h r} \approx$ $9.5 \Omega$, which is higher than the threshold for the longitudinal microwave instability.

\section{COHERENT SYNCHROTRON RADIATION}

Coherent synchrotron radiation (CSR) can cause emittance blow up and microwave instability [5]. Typically the beam is unstable only in an intermediate frequency range, namely above the beam-pipe shielding cut off and below the threshold frequency for Landau damping, if such a regime exists.

A novel code was recently developed [6] to calculate CSR effects in a storage ring over many turns. The shielding is computed from the actual vacuum chamber boundaries (no 'parallel-plate approximation'). At the moment only longitudinal CSR effects are included and CSR is treated only for the arc dipoles, not for the wigglers. However, it has been argued that the wiggler contribution is small [5]. The calculation uses a paraxial approximation, and the bunch shape is assumed not to change during the passage through a bending magnet (it does change from turn to turn under the influence of the CSR). Transient CSR components are automatically included and they are important for CLIC.

The initially Gaussian bunch is deformed under the influence of the CSR wake, shown in Fig. 1. The rms bunch 
length increases with increasing bunch charge. Figure 2 illustrates that for a beam-pipe radius of $2 \mathrm{~cm}$ the CSR microwave instability threshold is reached at about twice the nominal charge. Above the threshold the energy spread is no longer constant. For a beam-pipe radius of $4 \mathrm{~cm}$ the threshold would be only $20 \%$ above the nominal charge. Further results can be found in [7].

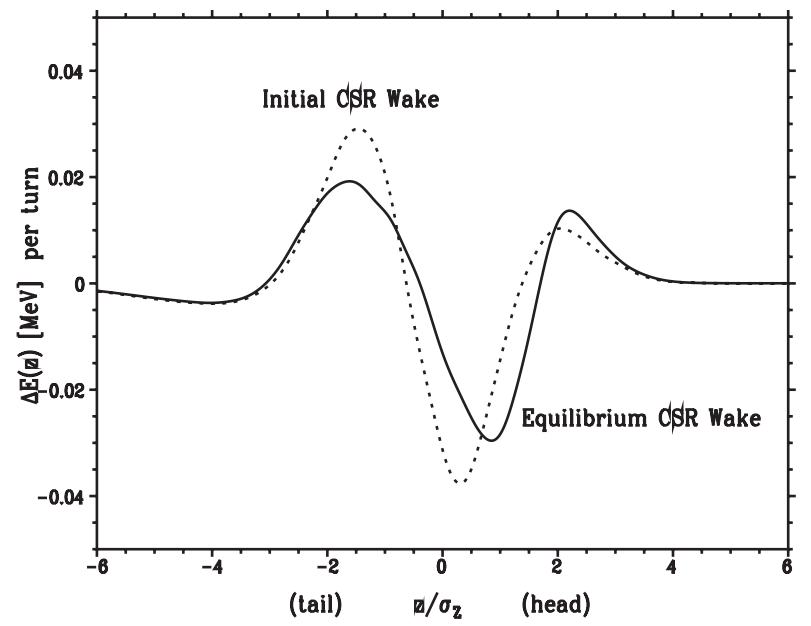

Figure 1: Initial (dashed) and equilibrium CSR wake (solid) of the CLIC damping ring for an arc beam pipe radius of $2 \mathrm{~cm}$ and a bunch population of $3 \times 10^{9}$.

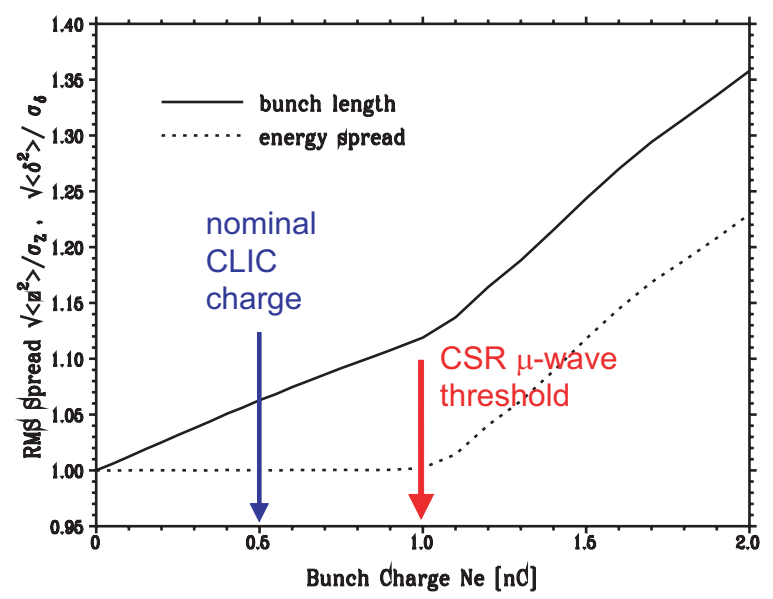

Figure 2: Rms bunch length (solid) and energy spread (dashed) as a function of bunch charge for an arc beampipe radius of $2 \mathrm{~cm}$.

\section{SPACE CHARGE}

The incoherent space charge tune shift is

$\Delta Q_{y}^{s c} \approx \frac{N_{b} r_{e} C}{(2 \pi)^{3 / 2} \gamma^{3} \sigma_{z}} \frac{1}{\sqrt{\epsilon_{x} \epsilon_{y}}}\left\langle\sqrt{\frac{\beta_{y}}{\beta_{x}+\frac{D_{x}^{2} \sigma_{\delta}^{2}}{\epsilon_{x}}}}\right\rangle \approx 0.11$,

which is close to the maximum acceptable value [8]. It could be reduced by raising the beam energy.

\section{ION INSTABILITIES AND ELECTRON CLOUD}

In the electron damping ring, at the end of a store the ion oscillation frequency inside the bunch train is about $1 \mathrm{GHz}$ for hydrogen, decreasing with $1 / \sqrt{A}$ for higher ion atomic masses $A$. After damping, the critical mass for trapping of a singly-charged ion is

$$
A_{\text {crit }}=\frac{N_{b} L_{\mathrm{sep}} r_{p}}{2 \sigma_{y}\left(\sigma_{x}+\sigma_{y}\right)} \approx 5 .
$$

Therefore, all ions except for hydrogen or helium are trapped by a bunch train. The ion-induced tune shift at the end of the train is

$$
\Delta Q_{\text {ion }} \approx \frac{N_{b} n_{b} r_{e} C}{\pi \sqrt{\left(\gamma \epsilon_{x}\right)\left(\gamma \epsilon_{y}\right)}}\left(\frac{\sigma_{\text {ion }} p}{k_{B} T}\right) \approx 0.014,
$$

where we assumed a pressure $p \approx 1$ ntorr, and an ionization cross section $\sigma_{\text {ion }}$ of 2 Mbarn (carbon monoxide or nitrogen). The exponential instability rise time of the fast beam-ion instabilty is

$$
\tau_{F B I I} \approx \frac{\gamma \sigma_{y} \sigma_{x}}{N_{b} n_{b} c r_{e} \beta_{y} \sigma_{\text {ion }}}\left(\frac{k_{B} T}{p}\right) \sqrt{\frac{8}{\pi}}\left(\frac{\sigma_{f_{i}}}{f_{i}}\right) \approx 3.3 \mu \mathrm{s} .
$$

This growth rate corresponds to about 3 turns, so that it could be controlled by a multi-bunch feedback system. Noise introduced by such a feedback may be a concern. A reduced vacuum pressure would ease the requirements.

Electron-cloud effects in the CLIC positron damping ring were discussed in $[9,10]$. In the arcs, antechambers absorb the entire photon flux. In the wiggler section, a residual photon flux of about $3 \times 10^{18} \mathrm{~m}^{-1} \mathrm{~s}^{-1}$ or about 3 photons per passing positron per meter length (about 30\% of the emitted ones) do not enter the antechamber. The average photon energy is about $2.2 \mathrm{keV}$. Simulated electron densities in the wiggler vary between $10^{13} \mathrm{~m}^{-3}$ and several $10^{14} \mathrm{~m}^{-3}$, which is to be compared with a simulated single-bunch instability threshold of about $2 \times 10^{12} \mathrm{~m}^{-3}$. This implies that special measures must be taken to reduce the electron density, such as the installation of dedicated photon stops intercepting the straight-ahead radiation, and the application of electric clearing fields.

\section{INTRABEAM SCATTERING AND TOUSCHEK LIFETIME}

Excitation by intrabeam scattering and radiation damping together determine the equilibrium emittance in the CLIC damping ring, with only a small additional contribution from quantum fluctuations. The equilibrium emittances with IBS are calculated using an iterative solution [11] based on the modified Piwinski formalism [12, 13]. The smallest transverse emittances are achieved with an rf voltage close to the energy loss per turn. This implies a small momentum acceptance, so that the lifetime of the stored beam is limited by the Touschek effect. The Touschek lifetime can be used as a diagnostics for emittance 
tuning and acceptance measurements [14, 15]. The Touschek lifetime can be computed using the Piwinski formalism of [16], including horizontal and vertical dispersion, which was implemented in the MAD-X programme [17]. Figure 3 illustrates how the Touschek lifetime, the transverse emittances, the bunch length, and the rms energy vary with the ring rf voltage, even for an rf voltage as low as 2.5 $\mathrm{MV}$, the Touschek lifetime is longer than the bunch-train store time of $90 \mathrm{~ms}$. A slight increase in rf voltage raises the beam lifetime substantially, which can be exploited for ring-tuning purposes.

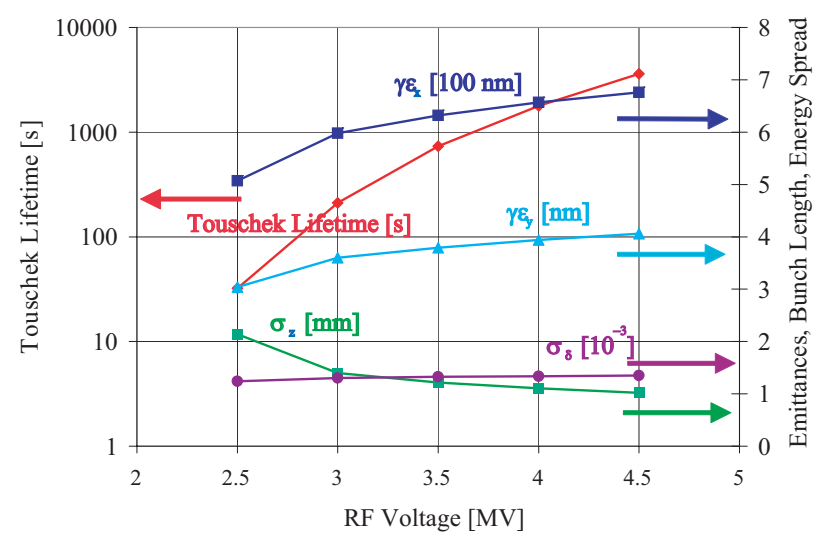

Figure 3: Touschek lifetime (red curve, left logarithmic scale) normalized transverse emittances, rms bunch length and momentum spread (other colors, right linear scale) as a function of $\mathrm{rf}$ voltage for a bunch population of $3.1 \times 10^{9}$, an rf frequency of $1.5 \mathrm{GHz}$, and an emittance coupling ratio of $0.6 \%$. The synchrotron-radiation energy loss per turn is $2.192 \mathrm{MeV}$.

\section{RESISTIVE WALL}

The dominant transverse impedance source is the resistive wall in the long wiggler sections with only about $8 \mathrm{~mm}$ vertical half aperture. The classical growth rate of the most unstable mode is estimated as

$$
\frac{1}{\tau_{r w}} \approx \frac{1}{2} \frac{\pi^{2}}{8} \frac{\beta_{y} N_{b} h r_{e} c^{2}}{2 \pi b_{w}^{3} \gamma \sqrt{\sigma c C}} \frac{1}{\sqrt{|Q-n|}} \approx 1700 \mathrm{~s}^{-1},
$$

where we have introduced the factor $\pi^{2} / 8$ to account for the flat chamber and another factor $1 / 2$, since the wigglers occupy about half the circumference. The parameter $h \approx 1785$ is the harmonic number. The ring was pessimistically assumed to be completely filled with $h$ equidistant bunches. Also, we have taken the resistivity of copper $\sigma \approx 5.4 \times 10^{17} \mathrm{~s}^{-1}$ and a fractional tune below the half integer, choosing $|Q-n| \approx 0.85$ for the most unstable coupled bunch mode. The classical resistive-wall growh time of $590 \mu$ s corresponds to about 500 turns.

\section{COUPLED-BUNCH INSTABILITIES}

Higher-order modes (HOMs) in the rf cavities could drive narrow-band transverse or longitudinal instabilities, as have been observed in many storage rings. These may be avoided by a careful design and dedicated HOM dampers. The average beam current in the CLIC damping ring is much lower than that reached at the two B factories.

\section{CONCLUSIONS}

The CLIC damping ring operates well below the longitudinal microwave and transverse mode-coupling thresholds. Coherent synchrotron radiation is benign, causing only a $5 \%$ bunch lengthening without instability. Intrabeam scattering was incorporated in the design optimization and the target emittances are reached including its effect. The Touschek lifetime is acceptable and can easily be increased for beam-tuning purposes, if desired. The resistive-wall instability driven by the impedance of the wiggler chamber can be suppressed by a feedback system. The space-charge tune shift is close to the limit considered acceptable. Potential limitations to be addressed are the high electron-cloud densities in the wiggler sections and the fast beam-ion instability. Possible remedies include clearing electrodes and photon stops for the wiggler, and an improved vacuum.

\section{ACKNOWLEDGEMENTS}

We thank H. Braun, H. Burkhardt, E. Metral, F. Ruggiero, E. Shaposhnikova and I. Wilson for helpful discussions and comments.

\section{REFERENCES}

[1] M. Korostelev, F. Zimmermann, Proc. Nanobeam'02 Lausanne, CERN-Proceedings-2003-001 (2001).

[2] A recent CLIC parameter optimization would lower both bunch charge and bunch spacing by $15 \%$, which does not affect our conclusions.

[3] D. Boussard, CERN Lab II/RF/Int 75-2 (1975); M.H. Wang, A. Chao, EPAC'04 Lucerne (2004).

[4] T. Ieiri et al., EPAC 2000, Vienna (2000).

[5] J. Wu et al., PRST-AB 6, 104404 (2003).

[6] T. Agoh, K. Yokoya, PRST-AB 7, 054403 (2004).

[7] T. Agoh, "Dynamics of Coherent Synchrotron Radiation by Paraxial Approximation,” Doctoral Thesis, U. Tokyo (2004).

[8] W. Decking, R. Brinkmann, EPAC 2000 Vienna (2000).

[9] D. Schulte, R. Wanzenberg, F. Zimmermann, ECLOUD'04 Napa (2004).

[10] F. Zimmermann, WIGGLE'05 Frascati (2005).

[11] M. Korostelev, F. Zimmermann, EPAC'02 Paris (2002).

[12] A. Piwinski, 9th HEACC'74 Stanford (1974).

[13] K.L.F. Bane, EPAC'02 Paris (2002).

[14] F. Zimmermann et al., ATF-98-10 (1998).

[15] T. Okugi et al., NIM A 455, 207 (2000).

[16] A. Piwinski, "The Touschek Effect in Strong Focusing Storage Rings,” DESY-98-179 (1998).

[17] C. Milardi, F. Schmidt, F. Zimmermann, http://mad.home.cern.ch/mad/touschek/touschek.html 